\title{
Could the use of nanowire structures overcome some of the current limitations of brain electrode implants?
}

\author{
"...in vitro, arrays of vertical nanowires promote neuronal \\ growth and limit glial cell spreading on the substrate."
}

First draft submitted: 25 January 2016; Accepted for publication: 3 February 2016; Published online: 16 March 2016

Keywords: brain implants $\bullet$ gliosis $\bullet$ nanostructures $\bullet$ nanowires $\bullet$ neural interfaces

\section{Current brain implants \& their limitations}

Brain electrode implants are expected to be a key enabling technology in Neuroscience, by helping deciphering how the brain works. It is also anticipated that brain implants will restore communication and/or motor functions in disabled patients.

The main challenge is to maintain a stable communication with the brain, in other words, maintaining steady signal amplitudes and recording from the same neurons over time. Such stable recordings are currently hampered by the movement of the probe in the tissue and/or the tissue foreign-body response to the implant. Indeed, current implants suffer from poor compatibility with the brain tissue, a feature mainly attributed to their inability to follow the movements of the brain tissue, but also to their large size and high stiffness. As a result, a decrease in neuronal density is observed near the implant, and glial cells encapsulate the implant, thereby introducing an insulating layer between the electrodes and the targeted neurons. Strategies to tackle this issue focus on reducing the size of implants, using soft materials in order to reduce the mechanical mismatch between brain tissue and the implant, and developing probes with high flexibility in 3D to improve tissue anchoring [1-3]. Another approach to improve the signal and reduce the tissue response has been to use electrodes with nanostructured surfaces [4], which can pos- sibly be combined with the above approaches to further increase the biocompatibility of brain implants.

\section{Vertical nanowire arrays as promising nanostructured surfaces} for brain implants

Among possible nanostructured surfaces, arrays of vertical nanowires show promising results for improving the biocompatibility of brain implants. Vertical nanowire arrays are arrangements of high aspect ratio nanostructures of 40-100 $\mathrm{nm}$ in diameter and $1-10 \mu \mathrm{m}$ in length, which are oriented perpendicularly to the substrate. In vitro, neurons cultured on gallium phosphide nanowire arrays show an increased neurite outgrowth compared with neurons cultured on standard culture substrates [5]. This rich and dense neurite network is thought to arise from neurotrophic biomolecules that would adsorb on the nanowires [5]. For instance, due to the high curvature of the nanowire surface, the cell adhesion-promoting protein laminin adsorbs up to four-times more per unit area on nanowires compared with flat substrates [6]. In a recent study, cryo-TEM images show that laminin indeed stretches out perpendicularly to the nanowires, resulting in the maximization of the number of proteins bound to the nanowires [7].

In contrast to neurons, glial cells cultured on vertical nanowire arrays were found to spread to a lesser degree compared with glial cells on standard culture substrates [5]. Fewer

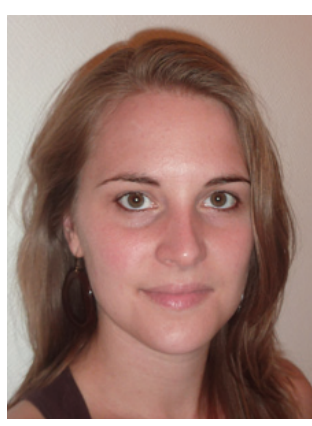

Gaëlle Piret

Clinatec laboratory, Biomedical Research Center Edmond J. Safra, INSERM/CEAléti/UJF/CHU, 38054 Grenoble Cedex 09, France

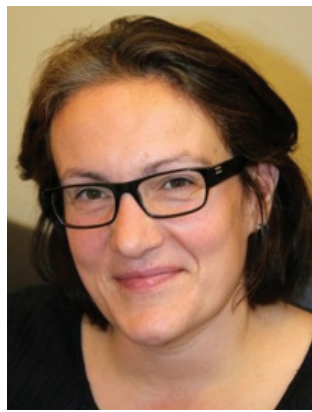

Christelle N Prinz

Author for correspondence: Division of Solid State Physics and NanoLund, Lund University, 22100 Lund, Sweden

and

Neuronano Research Center, Lund University, 22381 Lund, Sweden Tel.: +46462 224796

Fax: +46462 223637

christelle.prinz@ftf.Ith.se 
GFAP-positive cells were found on nanowire arrays compared with on flat substrates and their morphology resembles the star-like morphology they adopt in vivo, as opposed to the flattened, polygonal GFAP-positive cells found on flat substrates [5,8]. Moreover, on substrates with alternating regions of vertical nanowires and regions with a flat topography, glial cells mostly adhere and grow on the flat regions [8]. These results, taken together show that, in vitro, arrays of vertical nanowires promote neuronal growth and limit glial cell spreading on the substrate. This opens up for applications in vivo, where nanowires on the surface of brain implants could possibly promote neuronal adhesion, survival and growth at the interface between the implant and the tissue, as well as may limit the glial cell attachment, spread and proliferation on the implant surface, although this would have to be verified experimentally in vivo.

$$
66
$$

\section{movements of brain tissue and to improve the anchoring of the implant."}

...nanowires are expected to easily follow

The mechanisms limiting the spread of glial cells on nanowire arrays are not clear. One possible explanation is that glial cells growing on vertical nanowire arrays have a lower rate of proliferation, as it has been shown to be the case for fibroblasts [9]. In this case, the difference in cell numbers on nanowires and flat regions of the substrate would arise from a random cell positioning upon seeding and subsequent cell proliferation on the flat regions only. Such a mechanism would not prevent glial cells from adhering on the implant in vivo but may limit glial cell proliferation at the implant, which is one component of the foreign body response. Testing the brain tissue response to a soft implant coated with vertical nanowires would allow us to evaluate the effect of nanowire coating. As of today, only free-floating nanowires have been tested in terms of brain-tissue response. Short nanowires $(\leq 2 \mu \mathrm{m})$ have been shown to elicit the same tissue response as control vehicle injections, as opposed to longer nanowires ( 5 and $10 \mu \mathrm{m}$ ), which elicited a chronic inflammation in the brain tissue [10].

\section{Anchoring tissue \& mechanical compliance}

In addition to a possible reduction of gliosis, nanowires could possibly anchor the implant in the tissue while being able to locally follow the tissue movements. In vitro, cell adhesion is increased on vertical nanowire arrays, possibly through incomplete nanowire endocytosis. Indeed, nanowires are $50-100 \mathrm{~nm}$ in diameter, which is in the size range for endocytozed objects and which, in the case of vertical arrays, is the dimension sensed by cells when contacting the array (the nanowires are perpendicular to the cell surface upon contact). Since the nanowires are attached to their substrate, the endocytosis cannot be completed and the nanowires are not internalized, explaining the observed presence of cell membrane around the nanowires (see the following paragraph). Although the nanowire material has a rather high Young's modulus $(\approx 150 \mathrm{GPa})$, the spring constant of the nanowires is on the order of $10 \mathrm{mN} / \mathrm{m}$, which is three orders of magnitude lower compared with standard metal microwires used for brain recordings and two orders of magnitude lower than newly developed multifunctional flexible microelectrodes [11,12]. Axons have been observed to bend nanowires in a $U$ shape without them breaking or detaching from their substrate [13]. Therefore, nanowires are expected to easily follow movements of brain tissue and to improve the anchoring of the implant.

\section{Can nanowires be used as electrodes in brain implants?}

In vitro studies have shown that the cellular membrane, including the one of neurons, forms a tight seal on vertical nanostructures and nanowires [9,14-16], which, in the case of neural recordings, would increase the electrical seal resistance and lead to improved signal recordings. Arrays of vertical nanowires have been used to record neuronal signals in vitro [17], in intracellular configuration (where either the nanowires gained access to the cell interior spontaneously, or the membrane was permeabilized by applying a short electroporation pulse through the nanowires). Vertical arrays of nanowires have also been shown to measure single unit action potentials in vivo in the rat cortex [18]. In both sets of experiment listed above, the nanowires are coated with a conductive metal and the whole surface is subsequently isolated from the medium/tissue except for the nanowire tip. Although the in vivo experiments consisted of acute recordings only, they demonstrate that using nanowires as passive recording components is possible for measuring single-unit spikes in vivo. This opens up for having nanowires at the surface of brain implants, in other words, not only as simple coatings but also as sensing components.

\section{Future perspective}

We see a great potential in the integration of vertical nanowires in future brain implants. For instance, the transfer of nanowires at the surface of biocompatible, soft implants $[2,19]$ could further help decreasing the inflammatory response to the implant. In vitro, studies have shown that the nanowire material, provided it is biocompatible, does not play a major role in the observed beneficial effects on neuronal growth [5] and 
that, instead, the nanowire geometry is important. This gives us the freedom to select optimal inert materials in the case of implants aiming at long-term communication with the brain. Alternatively, high aspect ratio nanostructures could be made of inert materials resisting corrosion, which opens for many possible ways to overcome the limitations of current brain implants [20].

\section{Acknowledgements}

The authors thank Maria-Thereza Perez, Jens Schouenborg and Blaise Yvert for fruitful discussions.

\section{References}

1 Canales A, Jia X, Froriep UP et al. Multifunctional fibers for simultaneous optical, electrical and chemical interrogation of neural circuits in vivo. Nat. Biotechnol. 33(3), 277-284 (2015).

2 Agorelius J, Tsanakalis F, Friberg A, Thorbergsson PT, Pettersson LME, Schouenborg J. An array of highly flexible electrodes with a tailored configuration locked by gelatin during implantation - initial evaluation in cortex cerebri of awake rats. Front. Neurosci. 9, 331 (2015).

3 Liu J, Fu T-M, Cheng Z et al. Syringe-injectable electronics. Nat. Nanotechnol.10(7), 629-636 (2015).

4 Moxon KA, Kalkhoran NM, Markert M, Sambito MA, McKenzie JL, Webster JT. Nanostructured surface modification of ceramic-based microelectrodes to enhance biocompatibility for a direct brain-machine interface. IEEE Trans. Biomed. Eng. 51(6), 881-889 (2004).

5 Piret G, Perez MT, Prinz CN. Neurite outgrowth and synaptophysin expression of postnatal CNS neurons on GaP nanowire arrays in long-term retinal cell culture. Biomaterials 34(4), 875-887 (2013).

6 Hammarin G, Persson H, Dabkowska AP, Prinz CN. Enhanced laminin adsorption on nanowires compared with flat surfaces. Colloids Surf. B Biointerfaces 122, 85-89 (2014).

7 Hjort M, Bauer M, Gunnarsson S et al. Electron microscopy imaging of proteins on gallium phosphide semiconductor nanowires. Nanoscale (2016) (In Press).

8 Piret G, Perez M-T, Prinz CN. Support of neuronal growth over glial growth and guidance of optic nerve axons by vertical nanowire arrays. ACS Appl. Mater. Interfaces. 7(34), 18944-18948 (2015).

9 Persson H, Købler C, Mølhave K et al. Fibroblasts cultured on nanowires exhibit low motility, impaired cell division, and DNA damage. Small 9(23), 4006-4016 (2013).

10 Gallentoft LE, Pettersson LME, Danielsen N, Schouenborg J, Prinz CN, Linsmeier CE. Size-dependent long-term tissue

\section{Financial \& competing interests disclosure}

$G$ Piret is financed by INSERM and the ERC starting grant Brain Micro Snooper. C Prinz is financed by the Swedish Research Council (VR), the Center for Nanoscience at Lund University NanoLund, the Crafoord Foundation, the Carl Trygger Foundation, and the Neuronano Research Center at Lund University. The authors have no other relevant affiliationsor financial involvement with any organization or entity with a financial interest in or financial conflict with the subject matter or materials discussed in the manuscript apart from those disclosed.

No writing assistance was utilized in the production of this manuscript.

response to biostable nanowires in the brain. Biomaterials 42 , 172-183 (2015).

11 Suyatin DB, Hällström W, Samuelson L, Montelius L, Prinz $\mathrm{CN}$, Kanje M. Gallium phosphide nanowire arrays and their possible application in cellular force investigations. J. Vac. Sci. Technol. B 27(6), 3092 (2009).

12 Hällström W, Lexholm M, Suyatin DB et al. Fifteenpiconewton force detection from neural growth cones using nanowire arrays. Nano Lett. 10(3), 782-787 (2010).

13 Prinz C, Hällström W, Mårtensson T, Samuelson L, Montelius L, Kanje M. Axonal guidance on patterned freestanding nanowire surfaces. Nanotechnology 19(34), 345101 (2008).

14 Santoro F, Dasgupta S, Schnitker J et al. Interfacing electrogenic cells with 3D nanoelectrodes: position, shape, and size matter. ACS Nano 8, 6713-6723 (2014).

15 Hanson L, Lin ZC, Xie C, Cui Y, Cui BX. Characterization of the cell-nanopillar interface by transmission electron microscopy. Nano Lett. 12, 5815-5820 (2012).

16 Berthing T, Bonde S, Rostgaard KR et al. Cell membrane conformation at vertical nanowire array interface revealed by fluorescence imaging. Nanotechnology 23(41), 415102 (2012).

17 Robinson JT, Jorgolli M, Shalek AK, Yoon M-H, Gertner RS, Park H. Vertical nanowire electrode arrays as a scalable platform for intracellular interfacing to neuronal circuits. Nat. Nanotechnol. 7(3), 180-184 (2012).

18 Suyatin DB, Wallman L, Thelin J et al. Nanowire-based electrode for acute in vivo neural recordings in the brain. PLoS ONE 8(2), e56673 (2013).

19 Ahnen I Von, Piret G, Prinz CN. Transfer of vertical nanowire arrays on polycaprolactone substrates for biological applications. Microelectron. Eng. 135, 52-56 (2015).

20 Piret G, Hebert C, Mazellier JP et al. 3D-nanostructured boron-doped diamond for microelectrode array neural interfacing. Biomaterials. 53, 173-183 (2015). 